\title{
Synthesis of Novel Types of Segmented Polyamineurea and Polyamine-Poly(ethylene oxide) Block Copolymer
}

\author{
Hiroyuki Koyo, Teiji Tsuruta, and Kazunori KataokA* \\ Department of Industrial Chemistry, Faculty of Engineering, \\ Science University of Tokyo, Shinjuku-ku, \\ Tokyo 162, Japan \\ * Department of Materials Science and Research Institute for Biosciences, \\ Science University of Tokyo, \\ 2641 Yamazaki, Noda City, Chiba 278, Japan
}

(Received July 14, 1992)

\begin{abstract}
Telechelic polyamine with secondary amino-end groups was synthesized by the anionic addition reaction of polyamine macromonomer [poly $\left(N, N^{\prime}\right.$-diethyl- $N$-(4-vinylphenethyl)ethylenediamine)], with $N, N^{\prime}$-diethyl-ethylenediamine. Polyamineurea (I) was synthesized in one step by reacting the telechelic polyamine with equimole of diphenylmethane diisocyanate (MDI), while polyamine-urea (II) was synthesized in two steps by reacting the polyamine with two moles of MDI and then with diamine. An alternating block copolymer was synthesized by addition reaction between the polyamine and diisocyanate-terminated poly(ethylene glycol) (PEG), while multiblock copolymers consisting of polyamine and PEG were synthesized by reacting diisocyanate-terminated polyamine and diisocyanate-terminated PEG with a diamine. The structural analyses of the block type copolymers obtained were carried out with GPC, NMR, FT-IR, and differential scanning calorimetry (DSC).
\end{abstract}

KEY WORDS Telechelic Polyamine/Polyamineurea / Polyamine-Poly(ethylene oxide) Block Copolymer /

Molecular design of new types of functional polymers having polyamine segments in main chain has been carried out by our research group starting from novel polyamine macromonomer ${ }^{1-3}$ as one component. Through the study done to investigate the feasibility of these polymers as biomaterials, we reported previously that polyamine-graft-poly(hydroxyethyl methacrylate) copolymers (HA) exhibited unique biomedical behavior when they came into contact with living cells such as blood platelets or lymphocytes. ${ }^{4-6}$ For instance, the HA copolymer surface can recognize lymphocyte subpopulations, $B$ cells and $T$ cells, in physiological $\mathrm{pH}$ range, allowing $\mathrm{HA}$ to have utility as adsorbent for cell separation. ${ }^{7}$ In the course of our studies, we noticed that polyamine macromonomer had unique be- havior toward $\mathrm{pH}$ change. ${ }^{8}$ One of the most significant features of the macromonomer is the considerably weaker basicity compared with that of the low molecular weight analog.

Another important finding was that the spatial structure of the polyamine macromonomer was converted, reversibly, to the compact random coil conformation from the highly protonated extended conformation in the vicinity of $\mathrm{pH}$ 7.0. The unique behavior was ascribed to the chemical structure of the polyamine macromonomer consisting of alternating sequences of polar (diamine) and nonpolar (diethylenephenylene) units, which may closely be related to the recognition capability of HA surface against lymphocyte subpopulations (B and $\mathrm{T}$ cells), because polyamine side chains in HA copolymer surely 
responds to the $\mathrm{pH}$ change in a similar way to the polyamine macromonomer. ${ }^{4}$

Unique feature of the synthetic route of polyamine macromonomer is that it forms through anionic addition reaction of amine with double bond of styryl group catalyzed by lithium amide. Thus, telechelic polyamine with secondary-amino groups at both chain ends can be made quantitatively from polyamine macromonomer, reacting with excess diamine compounds. This telechelic polyamine with alkylated diamine units situated in the backbone (so-called integral type), is quite interesting chemicals to build up polyurea or polyurethane with integral diamine units through chain extending reaction with diisocyanate compounds, which may open a new area of application for segmented copolymers with $\mathrm{pH}$ or ion sensitive properties. This paper reports the preparation of novel segmented polyamineurea and polyamineurethaneurea with integral diamine units, starting from the newly designed telechelic polyamine with alternating units of hydrophobic and hydrophilic moieties.

\section{EXPERIMENTAL}

Most of the experiments were carried out under a purified nitrogen atmosphere to preclude oxygen and atmospheric moisture.

\section{Materials}

1,4-Divinylbenzene (supplied by Asahi Chemical Co.) was purified by fractional distillation over calcium hydride in vacuo. Commercial $N, N^{\prime}$-diethylethylenediamine was purified by two subsequent distillations over calcium hydride in vacuo, bp $69^{\circ} \mathrm{C}$ at $60 \mathrm{mmHg}$. Butyllithium was used as a cyclohexane solution and the concentration of the solution was determined by double titration. Other reagents and solvents were purified by standard methods and carefully distilled before use.

\section{Synthesis of Telechelic Polyamine}

$N, N^{\prime}$-Diethyl- $N$-(4-vinylphenethyl)ethylenediamine (1) was prepared as described previously. ${ }^{9,10}$ To a THF solution $(125 \mathrm{ml})$ of butyllithium $(14.6 \mathrm{mmol})$ and diisopropylamine (14.6 mmol), 1 (182.7 mmol) was added and allowed to stand in a thermostated bath at $20^{\circ} \mathrm{C}$. After $24 \mathrm{~h}$ reaction, the reaction mixture was added to a THF solution $(60 \mathrm{ml})$ containing reaction products from $N, N^{\prime}$ diethylethylenediamine (182.7 $\mathrm{mmol}$, DEDA) with butyllithium (14.6 mmol) (see Scheme 1). After $48 \mathrm{~h}$ reaction, the reaction was ceased by
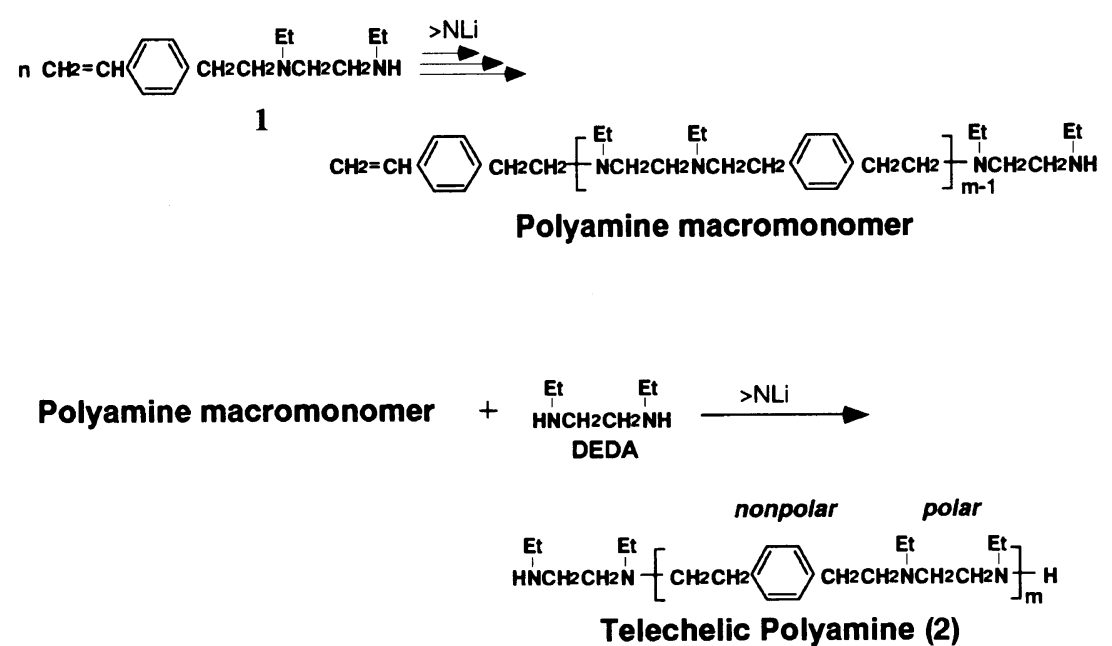

Scheme 1. 
adding a small amount of water. After precipitated lithium hydroxide was filtered off, the solvent and other low boiling point materials were removed by evaporation. The residual product was washed three times with a large excess of DMF, and was dried in vacuo to leave a colorless semisolid. The residual product was shown to be a telechelic polyamine (2) by analyses with UV, NMR, and FT-IR. For the measurement of molecular weight of 2, the double wavelength UV method with labeling agent [1-dimethylaminonaphthalene5-sulfonyl chloride (dansyl chloride)] was used. $^{11}$

\section{One-Step Method for Polyamineurea (I)}

Polyamineurea (I) was prepared by the addition reaction of 2 with $\mathrm{MDI}$ in $\mathrm{N}, \mathrm{N}$ dimethylacetamide (DMAc)/THF solution at room temperature, in a four-necked roundbottomed flask equipped with a stirrer and a dropping funnel (see Scheme 2). The molar ratio of 2 and MDI was $1: 1$. All experiments were carried out with $5 \mathrm{wt} \%$ solutions of 2 and of MDI. After $48 \mathrm{~h}$ reaction, the polymer obtained was precipitated in water-methanol [3:7 (v/v), $\mathrm{pH} 8.5]$, and then dried in vacuo for $48 \mathrm{~h}$. The product was analyzed by GPC, NMR, and FT-IR.

${ }^{1} \mathrm{H}$ NMR data $\left(500 \mathrm{MHz}\right.$, in $\left.\mathrm{C}_{6} \mathrm{D}_{6}\right): \delta=$
$0.9-1.1$ (multiplet, methyl protons in polyamine), $\delta=2.4-2.9$ (multiplet, methylene protons in polyamine), $\delta=3.8$ (singlet, methylene protons in MDI), $\delta=6.9-7.3$ (multiplet, aromatic protons in polyamine), $\delta=7.2-7.4$ (doublet, aromatic protons in MDI), $\delta=10.2$ (singlet, urea protons).

\section{Two-Step Method for Polyamineurea (II)}

The two-step method is shown in Scheme 2. The prepolymer was prepared by the reaction of 2 with MDI in DMAc/THF at room temperature. After the reaction, the mixture was cooled down to approximately $4^{\circ} \mathrm{C}$, and $5 \mathrm{wt} \%$ solution of diamine, 1,2-propylenediamine (PDA) or ethylenediamine (EDA), was added to the flask from a dropping funnel. The molar ratio of MDI, 2 and diamine was $2: 1: 1$. The polymer obtained was precipitated in water-methanol $[3: 7(\mathrm{v} / \mathrm{v}), \mathrm{pH} 8.5]$ and then dried in vacuo for $48 \mathrm{~h}$. The products were analyzed by elemental analysis and FT-IR.

\section{One-Pot Method for Polyamineurea Copolymer}

(I)

The one-pot method for polyamineurea copolymer (I) synthesis is shown in Scheme 3. An isocyanate-terminated PEG prepolymer was prepared in bulk by the reaction of PEG $\left(M_{n}=2018\right)$ with MDI (in 1:2 mole ratio) at

\section{One-step method}

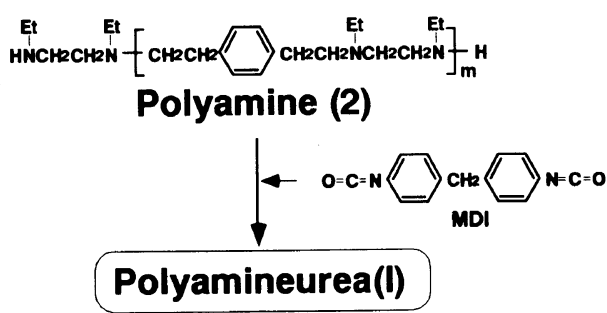

\section{Two-step method}

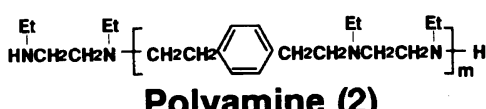

Polyamine (2)
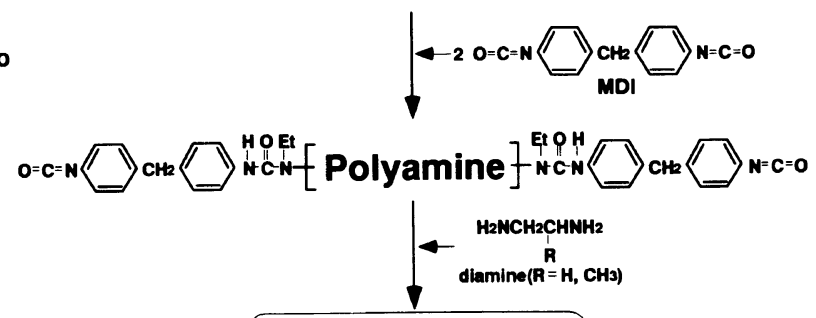

Polyamineurea(II)

Scheme 2. 
One-pot method

$\left.\mathrm{HO}-\mathrm{CH}_{2} \mathrm{CH}_{2} \mathrm{O}\right]_{n} \mathrm{H}$

Polyethyleneglycol(PEG)

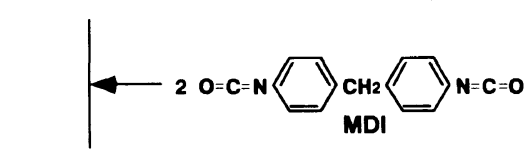

Prepolymer

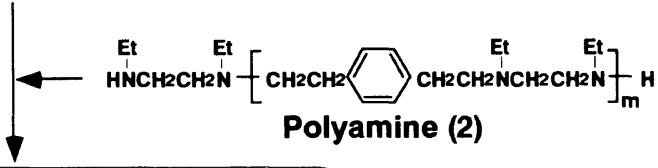

Polyamineurea copolymer(I)
Two-pot method

$X \underset{\mathrm{HNCH}_{2} \mathrm{CH} 2 \mathrm{~N}}{\mathrm{Et}}\left[\mathrm{CH}_{2} \mathrm{CH}_{2} \longrightarrow \mathrm{CH}_{2} \stackrel{\mathrm{CH}_{2} \mathrm{NCH}_{2} \mathrm{CH}_{2} \mathrm{~N}}{\mathrm{Et}}\right]_{m}^{\mathrm{H}}$

Polyamine (2)

Prepolymer(1)

Prepolymer(2)

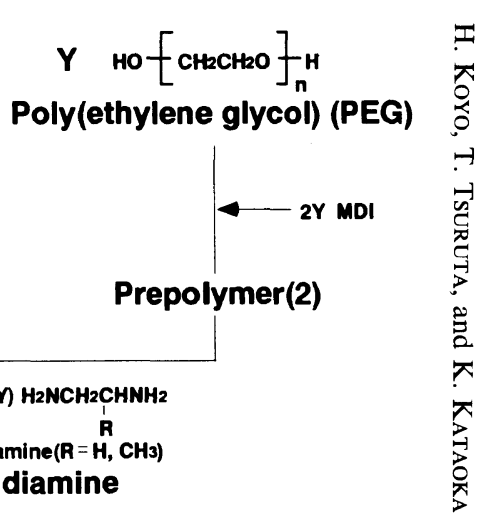

Polyamineurea copolymer(II)

Scheme 3. 
$80^{\circ} \mathrm{C}$. After $2 \mathrm{~h}$ reaction, the prepolymer was cooled down to approximately $4^{\circ} \mathrm{C}$, whereupon a DMAc-THF (4:1) mixture was added, and then $5 \mathrm{wt} \%$ solution of 2 in DMAc/THF was added from a dropping funnel. The molar ratio of PEG to 2 was $1: 1$. The reaction proceeded in a homogeneous system throughout the reaction time. The polymer formed was precipitated in ether and then dried in vacuo for $48 \mathrm{~h}$. The product was analyzed by GPC, NMR, and FT-IR.

${ }^{1} \mathrm{H}$ NMR data $\left(500 \mathrm{MHz}\right.$, in $\left.\mathrm{C}_{6} \mathrm{D}_{6}\right)$ : $\delta=1.0-1.1 \quad$ (multiplet, methyl protons in polyamine), $\delta=2.4-2.9$ (multiplet, methylene protons in polyamine), $\delta=3.4-3.6$ (singlet, methylene protons in PEG) $\delta=3.8$ (singlet, methylene protons in MDI), $\delta=4.25$ (singlet, PEG end protons adjacent to urethane groups), $\delta=6.9-7.3$ (multiplet, aromatic protons in polyamine), $\delta=7.4-7.6$ (doublet, aromatic protons in MDI), $\delta=10.2$ (singlet, urea plus urethane protons).

\section{Two-Pot Method for Polyamineurea Copolymer}

Polyamineurea copolymer (II) having different composition of polyamine and poly(ethylene oxide) segments were synthesized as shown in Scheme 3. Isocyanate-terminated prepolymers of polyamine and of PEG were prepared, respectively, as described above. After mixing the two prepolymers, $5 \mathrm{wt} \%$ solution of a diamine in DMAc/THF was added from a dropping funnel. The reaction system was found to keep its homogeneity. The polymer formed was precipitated in ether and methanol, and then dried in vacuo for $48 \mathrm{~h}$. The product was analyzed by FT-IR.

\section{Preparation of Polymer Films}

Transparent films were obtained by casting the polymer from DMAc solution. Characterization of the obtained polymer films was carried out by FT-IR and DSC measurement.

\section{Measurements}

A gel permeation chromatograph equipped with TSK gel G4000, G3000, and G2500 (Toyo Soda Co.) as columns was used. Products were detected with a differential refractometer (Shodex RI SE-31, Showa Denko Co.) and a Hitachi variable wavelength UV monitor. The elution/molecular weight calibration curves of products were plotted by making use of the polystyrene standard calibration curve. ${ }^{1} \mathrm{H}$ NMR spectra were measured in benzene with a JEOL Model GSX-500 spectrometer. For FT-IR measurement, JEOL JIR-5300 was used. UV measurements were recorded on a Hitachi 557 double wavelength-double beam spectrophotometer. Thermal properties of polymer films were determined by using a differential scanning calorimeter (DSC, Mettler TA 3000-DSC 30 system) at a heating rate of $20 \mathrm{~K} \mathrm{~min}^{-1}$ from $-120^{\circ} \mathrm{C}$ to $100^{\circ} \mathrm{C}$.

\section{RESULTS AND DISCUSSION}

\section{Telechelic Polyamine Having Secondary Amino- Group at the Chain Ends}

It was the prerequisite condition to start with a telechelic polyamine having a controlled molecular weight for the synthesis of a structurally controlled block type polyamineurea (or urethaneurea) consisting of desired length of polyamine segments in the polymer main chain. As reported ${ }^{3}$ previously, it was possible to control the molecular weight of the polyamine macromonomer by adjusting the concentration of lithium diisopropylamide in the initial mixture for the polyaddition reactions of 1 . The reaction mixture of the polyaddition reaction was subsequently used for the one-pot amination reaction with DEDA to obtain telechelic polyamine $2 .^{11}$

Results of UV analysis of the reaction mixture toward vinylphenethyl groups showed more than $99 \%$ of the polyamine chain ends to have reacted with DEDA. After prescribed treatment, product was obtained as colorless semisolid, in which the NMR signal of vinyl- 


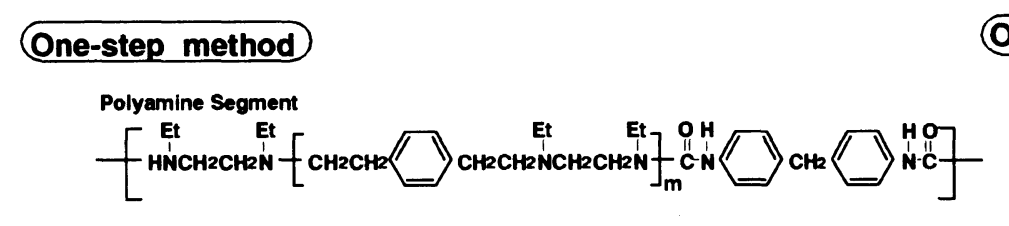

\section{Two-step method}

Polyamine Segment

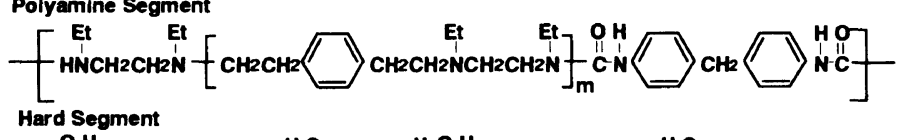
ard Segment

\section{One-pot method}

Polyamine Segment

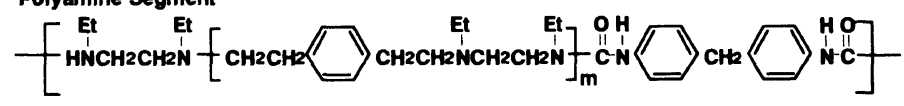

Poly(ethylene oxide) Segment

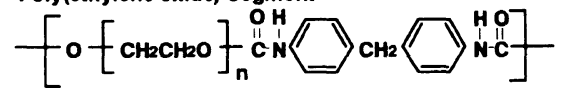

\section{Two-pot method}

Polyamine Segment

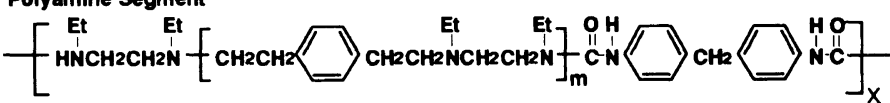

Poly(ethylene oxide) Segment

$\left[0-\left[\mathrm{CH}_{2} \mathrm{CH}_{2} \mathrm{O}\right]_{n}\right.$

$$
\text { - CN }
$$

ค

$\mathrm{R}=\mathrm{H}, \mathrm{CH} 3$

Scheme 4. 
phenethyl groups was not observed. From these measurements, the product was confirmed to be telechelic polyamine 2 shown in Scheme 1. From UV measurement of $\mathbf{2}$ with dansyl chloride, the number average molecular weight $\left(M_{n}\right)$ was determined $1850(m=7$ in Scheme 1).

\section{Synthesis of Polyamineurea}

Two types of polyamineurea were synthesized by using 2 as described in the experimental part. In the one-step method, 2 was reacted directly with MDI to form polyamineurea (I). In the two-step method, on the other hand, 2 was preliminarily converted to (or propylenediamine) to form polyamineurea (II). The structural formulas of polyamineurea (I) and (II) are shown in Scheme 4.

At the beginning, we looked for the most appropriate conditions for carrying out reactions between $\mathbf{2}$ and MDI. After several trials, we found a suitable reaction conditions under which the addition reactions proceeded in homogeneous solution throughout the reaction time. It seems that concentration of polyamine as well as the solvent composition have crucial effects on the solubility of reaction mixtures.

Polyamineurea (I): Results of polyamineurea (I) synthesis are shown in Table I. After $48 \mathrm{~h}$ reaction, the product formed was precipitated in water-methanol $(3: 7, \mathrm{v} / \mathrm{v}, \mathrm{pH}$ 8.5 ), and then dried in vacuo for $48 \mathrm{~h}$. The product was obtained in $85-92 \%$ yield with respect to the weight of 2 and MDI. Lower molecular weight products were probably washed away by the water-methanol mixture. The product was analyzed by GPC, NMR, and FT-IR.

From the GPC measurement shown in Figure 1, the number-average molecular weight $\left(M_{n}\right)$ and the polydispersity $\left(M_{w} / M_{n}\right)$ of the product were estimated to be $2.1 \times 10^{4}$ and 2.9, respectively.

The ${ }^{1} \mathrm{H}$ NMR data for polyamineurea (I) are shown in the experimental part, in which a singlet signal at $\delta=3.8$ is assignable to the methylene of MDI, while multiplet signals appearing at $\delta=2.4-2.9$ are to the 6 methylene groups adjacent to nitrogen in the diamine moiety of the polyamine. Integral ratio of the two types of methylene signal was estimated to be $1 / 39$, while the calculated value is $1 / 42$ for $m=7$ ( $c f$. Scheme 4). A signal at $\delta=10.2$ is assignable to amide (urea) protons, the intensity of which is observed to be just the same as that of MDI methylene protons. Signals of aromatic protons of MDI and

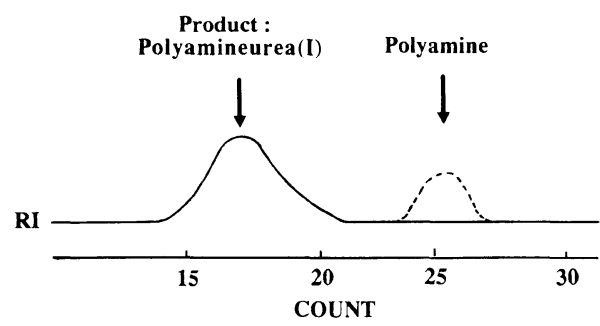

Figure 1. GPC chart of a polyamineurea (I) sample (run 3 in Table I).

Table I. Synthesis of polyamineureas $(\mathbf{I})^{\mathbf{a}, \mathbf{b}}$

\begin{tabular}{|c|c|c|c|c|c|c|}
\hline \multirow{2}{*}{ Run } & \multirow{2}{*}{$\frac{\text { Polyamine (2) }}{\mathrm{mmol}}$} & \multirow{2}{*}{$\frac{\text { MDI }}{\mathrm{mmol}}$} & \multirow{2}{*}{ DMAc/THF } & \multirow{2}{*}{$\begin{array}{c}\text { Yield } \\
\%\end{array}$} & \multirow{2}{*}{$M_{n}$} & \multirow{2}{*}{$M_{w} / M_{n}$} \\
\hline & & & & & & \\
\hline 1 & 1.45 & 1.44 & $1 / 1$ & 92.2 & 2.3 & 2.9 \\
\hline 2 & 1.39 & 1.40 & $3 / 1$ & 91.3 & 2.3 & 3.1 \\
\hline 3 & 1.65 & 1.66 & $4 / 1$ & 85.5 & 2.1 & 2.9 \\
\hline 4 & 1.75 & 1.75 & $4 / 1$ & 86.5 & 1.9 & 3.3 \\
\hline
\end{tabular}

a The concentrations of solutions of polyamine and MDI were $5 \mathrm{wt} \%$ each.

b Reaction time, $48 \mathrm{~h}$. 


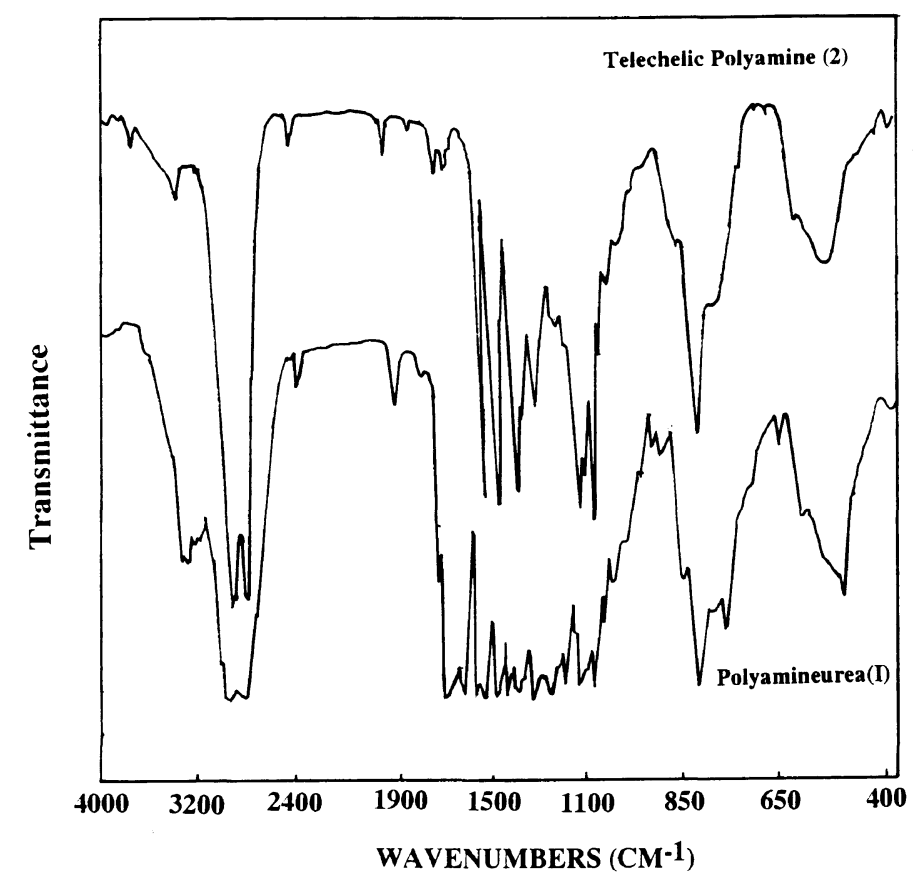

Figure 2. FT-IR spectra of polyamineurea (I) and telechelic polyamine (2).

Table II. Synthesis of polyamineureas (II)

\begin{tabular}{|c|c|c|c|c|c|c|c|}
\hline \multirow{2}{*}{ Run } & Polyamine (2) & MDI & Concn. & PDA & EDA & \multirow{2}{*}{ DMAc/THF } & \multirow{2}{*}{$\begin{array}{c}\text { Yield } \\
\%\end{array}$} \\
\hline & $\mathrm{mmol}$ & $\mathrm{mmol}$ & $w t \%$ & $\mathrm{mmol}$ & $\mathrm{mmol}$ & & \\
\hline 1 & 1.46 & 2.93 & 5 & 1.46 & & $3 / 1$ & 87.5 \\
\hline 2 & 1.71 & 3.43 & 5 & 1.71 & & $4 / 1$ & 88.7 \\
\hline 3 & 1.56 & 3.16 & 5 & 1.56 & & $4 / 1$ & 93.0 \\
\hline 4 & 1.62 & 3.29 & 5 & & 1.62 & $4 / 1$ & 97.0 \\
\hline 5 & 1.54 & 3.08 & 5 & & 1.54 & $4 / 1$ & 91.3 \\
\hline
\end{tabular}

polyamine are observed, respectively, at $\delta=7.2-7.4$ and $\delta=6.9-7.3$, and the integral ratio of the two types of aromatic protons was estimated to be $8 / 2$ in comparison with the calculated value $7 / 2$ ( $c f$. Scheme 4 ).

The FT-IR spectrum (Figure 2) of a film of the product shows characteristic bands at $1200-1300 \mathrm{~cm}^{-1}, 1680 \mathrm{~cm}^{-1}$, and $1720 \mathrm{~cm}^{-1}$ due to urea bonds, the former is assignable to the absorption band of hydrogen-bonded urea linkages in the polymer film. The spectrum of telechelic polyamine (2) is also shown in Figure 2 for comparison.
From these results, we conclude that the product possesses the structure of polyamineurea as shown in Scheme 4. We call this product as polyamineurea (I). The molecular weight of this particular polyamineurea (I) indicates 10 units of polyamine segments to be incorporated into the main chain.

Polyamineurea (II): Results of polyamineurea (II) synthesis are shown in Table II. After a similar treatment to that for polyamineurea (I), a polymeric product was obtained in $87-97 \%$ yield with respect to the weights of 2, MDI and ethylenediamine (or propylene- 
Synthesis of Novel Types of Segmented Polyamineurea and Polyamineurethaneurea

Table III. Solubility tests of polyamine and polyamineureas ${ }^{\mathrm{a}}$

\begin{tabular}{lccccc}
\hline \multicolumn{1}{c}{ Sample } & MeOH & Benzene & THF & DMF & DMAc \\
\hline Polyamine (2) & 0 & 0 & 0 & $\times$ & $\times$ \\
Polyamineurea (I) & $\bigcirc$ & $\bigcirc$ & $\bigcirc$ & $\times$ & $\bigcirc$ \\
Polyamineurea (II)-PDA & $\times$ & $\times$ & $\times$ & $\times$ & $\bigcirc$ \\
Polyamineurea (II)-EDA & $\times$ & $\times$ & $\times$ & $\times$ & \\
\hline
\end{tabular}

a Solubility tests were carried out at $25^{\circ} \mathrm{C}$ with $5 \mathrm{ml}$ of a solvent containing $0.1 \mathrm{~g}$ of polyamine or polyamineurea.

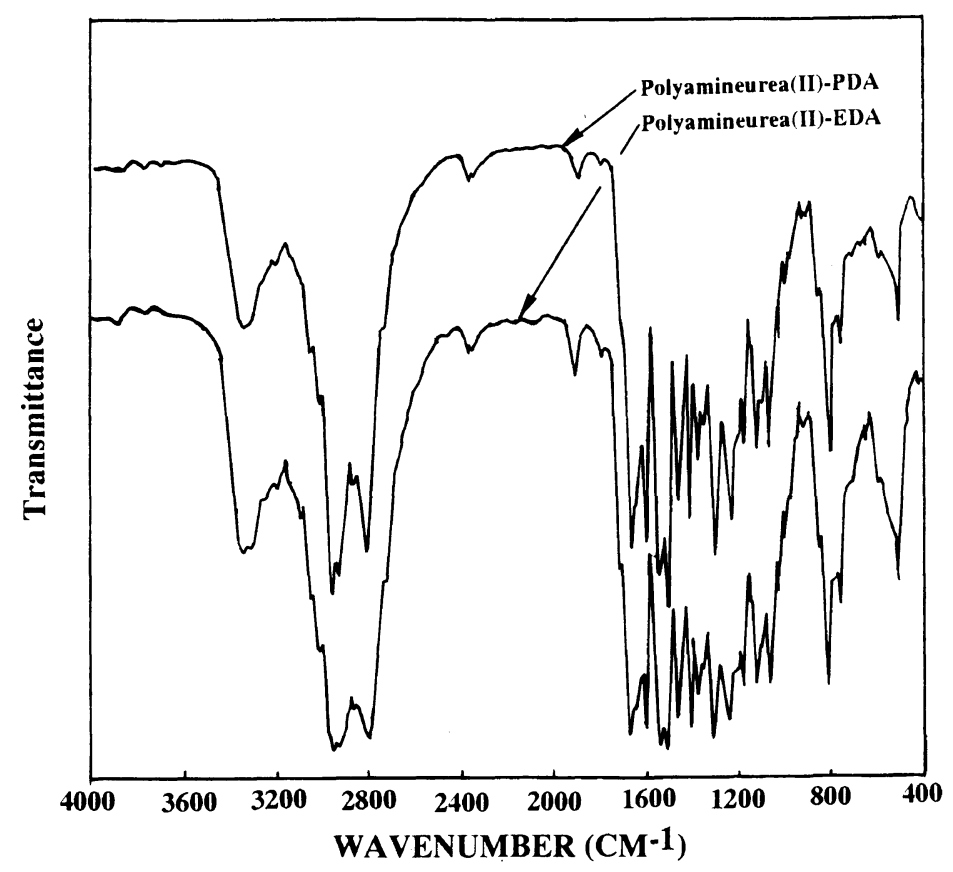

Figure 3. FT-IR spacta of polyamineurea (II)-EDA and-PDA.

diamine). The products were found to be sparingly soluble substances as shown in Table III. This may be explained by the presence of hard segments which are introduced by chain extending reactions with ethylenediamine (or propylenediamine). The FT-IR spectrum of polymer obtained showed the characteristic bands $1200-1300 \mathrm{~cm}^{-1}, 1650 \mathrm{~cm}^{-1}$, and 1720 $\mathrm{cm}^{-1}$ due to urea linkages (Figure 3). Further characterization of the product was not carried out owing to its poor solubility in organic solvents.
Polyamineurea Copolymer [Polyamine-Poly(ethylene oxide) Block Copolymer]

To improve the poor solubility of the segmented polyamineurea, we introduced poly(ethylene oxide) (PEO) segments into the main chain according to the two methods described in the experimental part.

In the one-pot method, an alternating copolymer of polyamine with PEG was synthesized by the reaction of isocyanateterminated prepolymer of PEG with 2 . In the two-pot method, on the other hand, a mixture of isocyanate-terminated prepolymers of $\mathbf{2}$ and 
H. Koyo, T. Tsuruta, and K. Kataoka

Table IV. Synthesis of polyamineurea copolymers (I) ${ }^{\mathrm{a}}$

\begin{tabular}{|c|c|c|c|c|c|c|c|c|c|}
\hline \multirow{2}{*}{ Run } & PEG & MDI & Polyamine (2) & Conen. & Temp & Time & Yield & $M_{n}^{\mathrm{b}}$ & \multirow{2}{*}{$M_{w} / M_{n}$} \\
\hline & $\mathrm{mmol}$ & $\mathrm{mmol}$ & $\mathrm{mmol}$ & $w t \%$ & ${ }^{\circ} \mathrm{C}$ & day & $\%$ & $\times 10^{-4}$ & \\
\hline 1 & 2.84 & 5.69 & 2.83 & 7 & R.T. & 4 & 86.5 & 1.7 & 3.1 \\
\hline 2 & 2.83 & 5.67 & 2.76 & 8 & R.T. & 9 & 92.1 & 2.6 & 3.3 \\
\hline 3 & 1.98 & 4.01 & 1.95 & 11 & 40 & 5 & 87.8 & 2.2 & 2.8 \\
\hline
\end{tabular}

a Reactions were carried out in DMAc-THF (4:1 in volume).

b Molecular weight was estimated by GPC.

of PEG were allowed to react with EDA (or PDA) to form a copolymer in which polyamine and PEO segments are randomly linked together via hard segments along the polymer chain. The alternating segmental polyamineurethaneurea is denoted by polyamineurea copolymer (I), while the randomly linked segmental one is denoted by polyamineurea copolymer (II).

Polyamineurea Copolymer (I): Results of polyamineurea copolymer (I) synthesis are shown in Table IV. After 4-9 day reaction, the product formed was precipitated in ether, and then dried in vacuo for $48 \mathrm{~h}$. The product was obtained in $86.5-92.1 \%$ yield. The product was analyzed by GPC, NMR, and FT-IR.

In the NMR spectrum of the product, a very sharp signal assignable to the methylene of PEO segments is observed at $\delta=3.4-3.6$ along with other signals at $\delta=3.8$ and $\delta=2.4-2.9$. The latter two signals were observed also in polyamineurea (I) as described above. A signal at $\delta=10.2$ is assignable to amide (urea plus urethane) protons, the intensity of which is observed to be just the same as that of MDI methylene protons. Integral ratio of methylene signals of PEO and the polyamine was 51/36. Considering the molecular weight of PEG (2018) and 2 (1850), the observed value for the integral ratio of the two signals at $\delta=3.4-3.6$ and $\delta=2.4-2.9$ is compatible with the alternating structure shown in Scheme 4. The FT-IR spectrum of polyamineurea copolymer

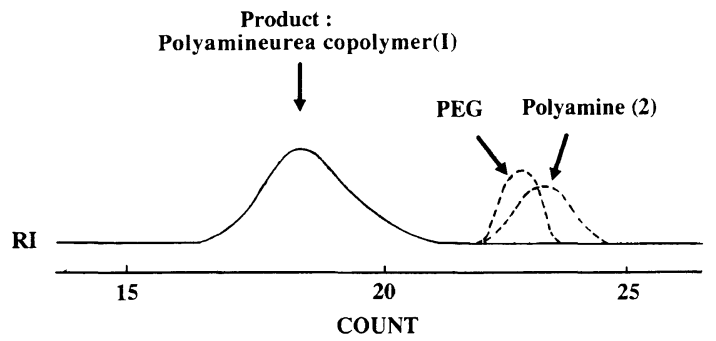

Figure 4. GPC chart of a polyamineurea copolymer (I) sample (run 1 in Table IV).

(I) film showed the characteristic bands at $1200-1300 \mathrm{~cm}^{-1}, 1650 \mathrm{~cm}^{-1}$, and $1720 \mathrm{~cm}^{-1}$ due to urea linkage.

The molecular weight of polyamineurea copolymer (I) was estimated to be $M_{n}=1.7 \times$ $10^{4}$ by GPC measurement as shown in Figure 4. This indicates that the polyamineurea copolymer (I) sample (Run 1) consists of four-time repetition of a polyamine-PEO sequence. Structural analyses of other samples of polyamineurea copolymer (I) were carried out similarly.

Polyamineurea Copolymer (II): Products formed by the two-pot method were precipitated in water-methanol $(3: 7=\mathrm{v} / \mathrm{v}, \mathrm{pH}$ 8.5 ), and then dried in vacuo for $48 \mathrm{~h}$ (Table V). The product was obtained in $85.1-92.3 \%$ yield. The product was analyzed by GPC, NMR, and FT-IR.

Polyamineurea copolymer (II) is a sparingly soluble substance as shown in Table VI, although it became soluble in DMF owing to the incorporation of $\mathrm{PEO}$ segments into the main chain (see Table III also). Molecular 
Synthesis of Novel Types of Segmented Polyamineurea and Polyamineurethaneurea

Table V. Synthesis of polyamineurea copolymers $(\mathbf{I I})^{\mathbf{a}, \mathbf{b}}$

\begin{tabular}{|c|c|c|c|c|c|c|c|c|c|}
\hline \multirow{4}{*}{ Run } & \multicolumn{2}{|c|}{ Prepolymer (1) } & \multicolumn{2}{|c|}{ Prepolymer (2) } & \multirow{4}{*}{$\frac{\mathrm{PDA}}{\mathrm{mmol}}$} & \multirow{4}{*}{$\begin{array}{c}\mathrm{EDA} \\
\mathrm{mmol}\end{array}$} & \multirow{4}{*}{$\frac{\text { Yield }}{\%}$} & \multirow{4}{*}{$\frac{M_{n}{ }^{\mathrm{c}}}{\times 10^{-4}}$} & \multirow{4}{*}{$M_{w} / M_{n}$} \\
\hline & \multirow{3}{*}{$\frac{\text { Polyamine }}{\mathrm{mmol}}$} & \multirow{3}{*}{$\begin{array}{l}\text { MDI } \\
\mathrm{mmol}\end{array}$} & \multirow{3}{*}{$\frac{\mathrm{PEG}}{\mathrm{mmol}}$} & \multirow{3}{*}{$\frac{\mathrm{MDI}}{\mathrm{mmol}}$} & & & & & \\
\hline & & & & & & & & & \\
\hline & & & & & & & & & \\
\hline 1 & 1.59 & 3.15 & 5.14 & 10.31 & 6.74 & & 87.1 & 2.15 & 2.22 \\
\hline 2 & 1.69 & 3.40 & 2.35 & 4.68 & 4.14 & & 92.3 & 1.93 & 2.73 \\
\hline 3 & 1.83 & 3.79 & 1.22 & 2.45 & 3.05 & & 85.1 & & \\
\hline 4 & 1.55 & 3.12 & 4.28 & 8.54 & & 5.83 & 92.1 & 2.43 & 2.20 \\
\hline 5 & 1.94 & 3.88 & 2.12 & 4.24 & & 4.06 & 90.0 & 1.92 & 2.35 \\
\hline 6 & 2.27 & 4.53 & 0.89 & 1.78 & & 3.16 & 86.0 & & \\
\hline
\end{tabular}

a The concentrations of solutions of polyamine and MDI were $5 \mathrm{wt} \%$ each.

b Reaction time, $48 \mathrm{~h}$.

c Determined by GPC (DMF as solvent).

Table VI. Solubility tests of polyamineurea copolymers (I) and (II) ${ }^{\mathrm{a}}$

\begin{tabular}{|c|c|c|c|c|c|c|c|c|}
\hline \multirow{2}{*}{ Sample } & \multicolumn{2}{|c|}{ Polyamine content } & \multirow{2}{*}{$\mathrm{MeOH}$} & \multirow{2}{*}{ Acetone } & \multirow{2}{*}{ THF } & \multirow{2}{*}{ Benzene } & \multirow{2}{*}{ DMF } & \multirow{2}{*}{ DMAc } \\
\hline & $\mathrm{mol} \%$ & wt $\%$ & & & & & & \\
\hline Copolymer (I) & & & 0 & 0 & 0 & 0 & 0 & 0 \\
\hline Copolymer (II)-24-PDA ${ }^{b}$ & 24.2 & 17.8 & $x$ & $x$ & $x$ & $x$ & O & 0 \\
\hline Copolymer (II)-42-PDA & 41.9 & 30.8 & $x$ & $x$ & $x$ & $x$ & 0 & 0 \\
\hline Copolymer (II)-60-PDA & 60.3 & 43.5 & $x$ & $x$ & $x$ & $x$ & $x$ & 0 \\
\hline Copolymer (II)-27-EDA & 27.1 & 19.7 & $x$ & $x$ & $x$ & $x$ & 0 & 0 \\
\hline Copolymer (II)-48-EDA & 48.2 & 35.5 & $x$ & $x$ & $x$ & $x$ & 0 & 0 \\
\hline Copolymer (II)-72-EDA & 71.8 & 54.1 & $x$ & $x$ & $x$ & $x$ & $x$ & 0 \\
\hline
\end{tabular}

a Solubility test were carried out at $25^{\circ} \mathrm{C}$ with $5 \mathrm{ml}$ of a solvent containing $0.1 \mathrm{~g}$ of polyamine or polyamineureas.

b Copolymer (II)-24-PDA, for instance, means the polyamine content to be $24 \mathrm{~mol} \%$ in copolymer (II) prepared with PDA as chain extender.

Table VII. Elemental analysis of polyamine copolymers (II)

\begin{tabular}{clllll}
\hline & & $\mathrm{N} / \%$ & $\mathrm{C} / \%$ & $\mathrm{H} / \%$ & $\mathrm{O} / \%$ \\
\hline Copolymer (II)-24-PDA & Calcd & 5.70 & 61.22 & 8.46 & 24.60 \\
& Found & 5.61 & 61.08 & 8.39 & 24.45 \\
Copolymer (II)-42-PDA & Calcd & 7.58 & 64.18 & 8.63 & 19.60 \\
& Found & 7.49 & 63.91 & 8.56 & 19.43 \\
Copolymer (II)-60-PDA & Calcd & 9.50 & 67.21 & 8.81 & 14.48 \\
& Found & 9.43 & 66.51 & 8.72 & 14.35 \\
Copolymer (II)-27-EDA & Calcd & 6.05 & 61.58 & 8.46 & 23.91 \\
& Found & 5.94 & 60.78 & 8.36 & 23.61 \\
Copolymer (II)-48-EDA & Calcd & 8.26 & 65.06 & 8.66 & 18.01 \\
& Found & 8.17 & 64.57 & 8.56 & 17.88 \\
Copolymer (II)-72-EDA & Calcd & 10.87 & 69.17 & 8.90 & 11.06
\end{tabular}


Table VIII. DSC of polyamineurea and polyamineurea copolymers

\begin{tabular}{llll}
\hline \multicolumn{1}{c}{ Sample } & $T_{\mathrm{g}} /{ }^{\circ} \mathrm{C}$ & $T_{\mathrm{m}} /{ }^{\circ} \mathrm{C}$ \\
\hline A & Polyamine (2) & -60.0 & \\
& Polyamineurea (I) & -29.8 & \\
& Polyamineurea (II)-PDA & -21.2 & \\
& Polyamineurea (II)-EDP & -23.0 & \\
\hline B Copolymer (I) & -41.7 & 36.3 \\
& PEO-PDA & -39.8 & 38.0 \\
Copolymer (II)-24-PDA & -33.9 & 43.6 \\
Copolymer (II)-42-PDA & $-35.1,-14.2$ & 36.3 \\
Copolymer (II)-60-PDA & -25.3 & 44.6 \\
PEO EDA & -41.2 & 38.5 \\
Copolymer (II)-27-EDA & $-42.1,-7.59$ & 28.9 \\
Copolymer (II)-48-EDA & $-39.9,-8.73$ & 28.8 \\
Copolymer (II)-72-EDA & $-40.0,-5.78$ & 28.0 \\
\hline
\end{tabular}

weight of polyamineurea copolymer (II) determined by GPC (DMF as solvent) are shown in Table V.

The FT-IR spectrum of polymer film showed the characteristic band at $1200-1300 \mathrm{~cm}^{-1}$, $1650 \mathrm{~cm}^{-1}$, and $1720 \mathrm{~cm}^{-1}$ due to urea bonds. The results of elemental analysis of polyamineurea copolymer (II) are listed in Table VII, where the calculated values were obtained by assuming the mole ratio in feed of polyamine-PEO-diamine to be kept in polyamineurea copolymer (II). As seen from Table VII, the above assumption was well supported by the analysis data, although three of them deviate to larger extents than experimental errors.

\section{DSC Behavior of Polymer Films}

Table VIII(A) shows glass transition temperature $\left(T_{\mathrm{g}}\right)$ of polyamine as well as polyamineurea (I) and (II). Films made of these polymers exhibited no endothermal peak corresponding to the melting temperature $\left(T_{\mathrm{m}}\right)$. The two types of polyamineurea (II) having hard segments show higher $T_{\mathrm{g}}$ compared with polyamineurea (I).
As shown in Table VIII(B), polyamineurea copolymers showed endothermal peak corresponding to the $T_{\mathrm{m}}$ of PEO segments, indicating that the PEO segments in these polymer films were partially crystallized. It was suggested from the $T_{\mathrm{m}}$ data that polyamineurea copolymer (II)-PDA has more distinct aggregation of PEO segments than that of copolymer (II)-EDA. Dual glass transition temperatures observed in copolymer (II) are presumably originated from the different structures of hard segments which can be urea-urea or urea-urethane or urethane-urethane enchainments.

Some of these polymers were found to exhibit high $\mathrm{pH}$-sensitivity in swelling-contraction behavior. Full details will be reported elsewhere.

\section{REFERENCES}

1. A. Maruyama, E. Senda, T. Tsuruta, and K. Kataoka, Makromol. Chem., 187, 1895 (1986).

2. T. Nishimura, M. Maeda, T. Tsuruta, and K. Kataoka, Makromol. Chem., Rapid Commun., 1, 573 (1980).

3. Y. Nabeshima, A. Maruyama, T. Tsuruta, and K. Kataoka, Polym. J., 19, 593 (1987).

4. A. Maruyama, T. Tsuruta, K. Kataoka, and Y. Sakurai, Biomaterials, 9, 471 (1988).

5. A. Maruyama, T. Tsuruta, K. Kataoka, and Y. Sakurai, J. Biomed. Mater. Res., 22, 555 (1988).

6. Y. Nabeshima, T. Tsuruta, K. Kataoka, and Y. Sakurai, J. Biomater. Sci., Polym. Ed., 1, 85 (1989).

7. K. Kataoka, in "Cell Separation Science and Technology” D. S. Kompala and P. Todd, Ed., ACS Symp. Ser. 464, American Chemical Society, Washington, D.C., 1991, p 159.

8. A. Maruyama, T. Tsuruta, K. Kataoka, and Y. Sakurai, Makromol. Chem., Rapid Commun., 8, 27 (1987).

9. Y. Nitadori and T. Tsuruta, Makromol. Chem., 180, 1877 (1979).

10. T. Nishimura, M. Maeda, N. Nitadori, and T. Tsuruta, Makromol. Chem., 183, 29 (1982).

11. S. Kitano, T. Tsuruta, and K. Kataoka, unpublished data. 